\title{
TV/Series
}

14 | 2018

Posthumains en séries

\section{« My own fucking story » : le raga de Westworld}

\section{Briac Picart-Hellec}

\section{OpenEdition}

\section{Journals}

Édition électronique

URL : http://journals.openedition.org/tvseries/3194

DOI : 10.4000/tvseries.3194

ISSN : 2266-0909

\section{Éditeur}

GRIC - Groupe de recherche Identités et Cultures

\section{Référence électronique}

Briac Picart-Hellec, « « My own fucking story » : le raga de Westworld», TV/Series [En ligne], 14 | 2018, mis en ligne le 31 décembre 2018, consulté le 19 avril 2019. URL : http://journals.openedition.org/ tvseries/3194; DOI : 10.4000/tvseries.3194

Ce document a été généré automatiquement le 19 avril 2019

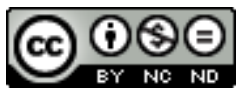

$T V / S e r i e s$ est mis à disposition selon les termes de la licence Creative Commons Attribution - Pas d'Utilisation Commerciale - Pas de Modification 4.0 International. 


\title{
« My own fucking story »: le raga de Westworld
}

\author{
Briac Picart-Hellec
}

Dans un entretien avec le jazzman Ornette Coleman, Jacques Derrida explique :

[...] le concept même de l'improvisation tient à la lecture, car ce que nous entendons souvent par improvisation, c'est la création de quelque chose de nouveau, mais qui n'exclut pas la trame préécrite, qui la rend possible ${ }^{1}$.

2 Dans un cours donné en 1981 à l'université Paris 8, Gilles Deleuze, lui, compare l'importance du «timing», le moment où un instrument fait son entrée dans une improvisation jazz, avec le concept grec du kairos, qu'il définit comme suit: «Le kaïros c'est : exactement le bon moment. Ne pas rater le bon moment. [...] Il y avait un Dieu, il y avait une espèce de puissance divine du kaïros chez les Grecs. L'occasion favorable, l'opportunité $[. . .]^{2}$. »

3 Nous avons donc deux regards sur l'improvisation: l'improvisation comme possible uniquement dans un cadre prédéfini, et l'improvisation comme acte libérateur, quasi divin. Cela n'aura échappé à aucun spectateur: Westworld (HBO, 2016-), est une série particulièrement musicale, notamment à travers ces plans récurrents d'un piano automatique reprenant des morceaux pop du vingtième siècle, que l'on peut voir dès le générique. Mais qu'est-ce qu'un piano, programmé pour reprendre un morceau à la note près, pourrait bien avoir à nous dire sur la notion d'improvisation?

«Some people choose to see the ugliness in this world. The disarray. I choose to see the beauty. To believe there is an order to our days, a purpose ${ }^{3}$.» Dès cette réplique prononcée dans sa première minute, Westworld, n'aura eu de cesse de diviser son univers. «Hosts» et "guests", chapeaux blancs et chapeaux noirs. Elle aura aussi foncièrement divisé son public. Si des critiques ont pu trouver la série brillante, d'autres l'ont, plus prosaïquement, traitée de «bullshit ${ }^{4}$ ». Dans les articles et les commentaires des spectateurs les plus négatifs, on pointe deux défauts clés. Le premier tiendrait à ce que les rebondissements et retournements (twists) de la série auraient été trop prévisibles: les scénaristes auraient sous-estimé la communauté internet qui a anticipé chaque 
retournement narratif et, par extension, la série aurait donc sous-estimé ses spectateurs ${ }^{5}$. Le deuxième tient en ce que la série serait trop froide sur le plan affectif. Parce que la plupart de ses héros sont des êtres dont le comportement suit un script, le spectateur ne pourrait s'impliquer dans leurs décisions puisqu'elles ne sont par nature pas les leurs; l'identification ne fonctionnerait donc $\mathrm{pas}^{6}$. Si ces spectateurs ont choisi de voir la confusion de Westworld, l'objectif de ce texte sera de tenter d'en voir l'ordre et le but. Ces défauts ne sont-ils pas au contraire en fait en parfaite adéquation avec le sens éthique du récit?

5 Commençons par le premier point, c'est à dire la prévisibilité des stratégies narratives de Westworld. Dans le chapitre intitulé "What's a story? » de son ouvrage On Filmmaking, Alexander Mackendrick avance une hypothèse pour expliquer notre besoin de fictions :

Psychotherapists will warn you that a figure in a dream should not always be thought of as a person in the same way that the waking mind conceives individuals. Dream-figures are more often personifications of some aspects of the dreamer's psyche. This could be a clue to the psychological purpose of all stories. A story that has fictional characters may be using these figments of the imagination or unconscious in order to act out an abstract thought, an idea, a theme [...]. A myth, it is said, is the verbal equivalent of rite that serves the same archaic need: to help the primitive mind take hold of a mystery. Stories, even in the contemporary context of mass entertainment, would seem to be successful when they, too, fulfil such a need, something audiences need not even be aware for?

La série nous signifie clairement, de par la répétition des termes "narrative », "backstory " ou «writer» dans ses dialogues, qu'elle ne traite pas seulement d'intelligence artificielle mais, plus réflexivement, de fiction. « Have you ever questioned the nature of your reality ${ }^{8}$ ? demande-t-on régulièrement à Dolores. Westworld semble ici demander à son spectateur : «As-tu déjà remis en question la nature de tes rapports aux personnages de fiction?» Celui-ci voit dans la série les invités perdre leur humanité en traitant les hôtes comme des objets, alors que, comme le dit Felix à Maeve : «We are the same these days ${ }^{9}$. "Westworld requiert donc que nous fassions un effort: si Felix peut comprendre qu'un être manufacturé pour le plaisir de ses semblables n'est pas moins réel et n'est pas moins une personne que lui, ne sommes-nous pas également capables d'accepter que des personnages de fiction créés pour nous divertir le soient eux aussi ? Dans le contexte de Westworld, la vision de la fiction qu'expose Mackendrick apparaît comme de l'exploitation pure et simple puisqu'elle correspond à la philosophie de l'horrible Logan lorsqu'il dit à William : "This place is the answer to the question that you've been asking yourself : who you really $\operatorname{are}^{10}$. " Attendre de la série qu'elle réponde à cette question, considérer ses personnages comme des extensions de notre psyché et non comme des individus à part entière, c'est se rendre coupable de la même réification que commettent les invités envers les hôtes. C'est, tout simplement, être un chapeau noir.

7 Mackendrick présente la fiction comme un procédé permettant de mieux appréhender le mystère de l'existence - la fiction comme un procédé initiatique et comme outil pour comprendre le but de nos existences, selon les termes de Dolores. L'idée de l'ordre sousjacent à la confusion renvoie à la fois à la chronologie fragmentée du récit et au labyrinthe d'Arnold. Comme on l'apprend dans le dernier épisode de la saison, le labyrinthe, avec ses tours et détours (twists and turns) n'a pas été conçu pour les invités mais pour les hôtes. Autrement dit, la narration tortueuse et complexe de Westworld, 
ses "twists ", son labyrinthe, ne nous étaient pas destinés à nous les spectateurs, mais bien aux personnages. Il a été reproché, à juste titre, aux twists de Westworld de manquer d'originalité : l'épisode «Ji Yeon » de LOST reposait déjà sur un rebondissement basé sur une double temporalité et, de même, la révélation que Bernard est un hôte n'aura pas surpris les spectateurs de Dollhouse. Mais Westworld avait déjà prévenu son public que son but n'était pas nécessairement l'originalité : de la même manière que les pianos du saloon reprennent des tubes du vingtième siècle, Westworld «rejoue les tubes" des grandes séries des années 2000. Compte tenu de son statut de remake, la série est elle-même une reprise. Le corpus intertextuel formé par ces références représente, si l'on suit la pyramide d'Arnold (mémoire, improvisation, intérêt personnel, esprit bicaméral), la mémoire de la série, mémoire sur laquelle elle s'appuie pour accéder à l'improvisation. De la même manière que c'est le but du labyrinthe et non sa forme qui importe (puisqu'il est en réalité, dans sa forme physique, un simple jouet pour enfant), la forme prise par les twists importe peu : ce qui compte c'est leur effet.

Arnold était animé par le désir de libérer ses créatures. Ce but n'a pas passionné certains spectateurs parce qu'ils ne pouvaient éprouver de l'empathie pour les hôtes, en raison de leur nature de personnages programmés. Cette raison compréhensible est cependant paradoxale, puisque c'est en partie ce qui, dans la diégèse, transforme William le romantique en sadique Homme en Noir. Au-delà de l'amnésie de Dolores, ce que William ne peut supporter c'est l'idée que leur rencontre - cette scène où il ramasse la boîte de conserve qu'elle vient de faire tomber - soit en réalité un événement programmé : que des dizaines d'autres invités l'aient vécu et que des centaines d'autres soient sur le point de le vivre à leur tour ${ }^{11}$. On pense ici aux fans d'un groupe de musique indépendant qui le renient dès que celui-ci perce chez le grand public: il peut en effet être difficile de comprendre que cette relation si singulière, si intime, avec ce qu'on aimait soit commune à beaucoup d'autres. Si certains y verront une salutaire forme de communion entre inconnus, d'autres comme William le vivront comme une trahison.

9 Dans sa précédente série Person of Interest (CBS, 2011-2016), Jonathan Nolan avait déjà souligné l'importance des flash-backs. À mesure que la série se déroulait, on découvrait peu à peu le passé des différents protagonistes, un peu comme dans LOST. Dans le dernier épisode, la machine créée par Harold Finch pour prédire les attentats expliquait son propre fonctionnement : « You built me to predict people, Harry. But to predict them, you have to truly understand them, and that proved to be very difficult indeed. So I began by breaking their lives down into moments. Trying to find the connections, the things that explained why they did what they did. ${ }^{12}$ " Nolan mettait à nu le procédé narratif de sa série, qui pour traiter de l'humanisation de la machine doit aussi nous permettre de comprendre les personnages humains, les flashbacks jouant un rôle essentiel dans le processus d'identification du spectateur. Comment comprendre alors que dans Westworld il nous demande de nous attacher à des personnages dont les souvenirs, exposés à travers des flashbacks, sont perpétuellement à remettre en question car artificiels?

Nolan nous rappelle là que les flashbacks dans une fiction sont toujours artificiels en ce qu'ils sont toujours écrits, donc programmés par le scénariste. Un personnage n'a pas de volonté propre et suit les désirs de son auteur. Il est étonnant que cette situation ne nous gêne que lorsqu'elle est explicitement affirmée aussi dans la diégèse. Un personnage est toujours un hôte à la merci d'un maître architecte et metteur en scène comme Ford ou pire, Lee Sizemore. C'est ce que la série cherche à souligner. La clé de la prise de conscience des hôtes repose justement sur un refus de se laisser dicter ce dont ils doivent 
se souvenir. « They can make you forget... Well I guess not you ${ }^{13}$ » dit Felix à Maeve. C'est cet état de fait, cette subordination des hôtes aux scénarios des humains, qui préoccupe Westworld.

11 Dans sa célèbre nouvelle « La Bibliothèque de Babel », Jorge Luis Borges décrit un monde constitué d'une gigantesque bibliothèque. Les livres qui la composent semblent de prime abord écrits dans un charabia incompréhensible que les habitants de la bibliothèque cherchent en vain à déchiffrer. Mais, comme le narrateur le révèle, un bibliothécaire a fini par comprendre le véritable sens du lieu :

[...] tous les livres, quelque divers qu'ils soient, comportent des éléments égaux: l'espace, le point, la virgule, les vingt-deux lettres de l'alphabet. Il fait également état d'un fait que tous les voyageurs ont confirmé : il n'y a pas dans la bibliothèque, deux livres identiques. De ces prémisses incontroversables, il déduisit que la Bibliothèque est totale, et que ses étagères consignent toutes les combinaisons possibles des vingt et quelques symboles orthographiques (nombre, quoique vaste, non infini), c'est-à-dire tout ce qu'il est possible d'exprimer dans toutes les langues ${ }^{14}$.

Ce concept n'a pas manqué récemment d'inspirer un programmeur nommé Jonathan Basile. Basile a en effet créé un algorithme fonctionnant sur le même principe que la bibliothèque de Borges, et qui génère toutes les possibilités de combinaison de 3200 caractères. On peut accéder à cet algorithme à travers un site divisé comme dans la nouvelle de Borges, c'est à dire en hexagones, murs et étagères remplis de volumes d'un maximum de 410 pages. Comme dans la nouvelle, on peut donc y trouver n'importe quel extrait de n'importe quelle œuvre littéraire. Le début de La Tempête de Shakespeare se trouve par exemple à la douzième page du dix-huitième volume de la troisième étagère du troisième mur d'un hexagone au nom bien sûr imprononçable puisqu'aléatoirement formé d'une suite de caractères. Mais le potentiel du site est beaucoup plus vaste.

Par exemple : virtuellement, les circonstances de la mort de chacune des personnes qui liront ces lignes y sont déjà inscrites. Mais on ne connaît pas leur emplacement dans la bibliothèque. Tout ce que chacun d'entre nous pourra jamais penser, dire, écrire, y est inscrit. Cette dernière phrase se trouve à la soixante-dix-huitième, du vingt-cinquième de la troisième étagère du quatrième mur d'un des hexagones. L'expérience de la visite de ce site rappellera au spectateur de Westworld l'une des scènes les plus puissantes de la série : celle de l'épisode " The Adversary » (S01E06) où Maeve voit son propre algorithme sur la tablette de Félix, anticipant chacune de ses réponses.

14 Ainsi, si l'on s'obstine à vouloir voir en Westworld la réponse à la question « Qui sommesnous?», il semble qu'un début de réponse apparaisse assez clairement. Lorsque Felix affirme à Maeve qu'il est humain, Maeve lui répond: «How do you know ${ }^{15}$ ?", apostrophant là les spectateurs, par-delà Felix lui-même : Avez-vous déjà remis en question la nature de votre réalité? Comment être sûr que vous n'êtes pas un personnage de fiction? Et si depuis le début vous n'étiez ni le pianiste, ni l'auditeur, mais le piano mécanique?

Tout comme pour Maeve, un algorithme a en effet déjà anticipé nos pensées, et nous sommes en un sens scriptés. Comme l'explique Ford à Bernard: «Every host needs a backstory, Bernard. You know that. The self is a kind of fiction for hosts and humans alike ${ }^{16}$.» Notre existence est surdéterminée par des facteurs préexistants à notre volonté : notre nationalité, notre classe sociale, notre sexe, nos limites physiques, les décisions politiques que nous impose le régime politique dans lequel nous vivons, etc. Il existe toujours des grands récits «cadres" (équivalent des «narratives» de Ford ou de Sizemore) auxquels l'on est censés se conformer: que ce soient les rôles de genre par 
exemple, ou le parcours social type (faire des études, trouver un emploi, se marier, acheter une maison, avoir des enfants, etc.). Comme les hôtes, nous n'avons qu'une petite marge de manœuvre, une infime capacité à improviser. Mais pour le reste, nous suivons le script ${ }^{17}$. C'est en substance ce que Ford explique à Bernard quand celui-ci lui demande : «So what's the difference between my pain and yours, between you and me ${ }^{18}$ ?»

FORD. This was the very question that consumed Arnold, filled him with guilt, and eventually drove him mad. The answer always seemed obvious to me. There is no threshold that makes us greater than the sum of our parts, no inflection point at which we become fully alive. We can't define consciousness because consciousness does not exist. Humans fancy that there's something special about the way we perceive the world, and yet we live in loops as tight and as closed as the hosts do, seldom questioning our choices, content, for the most part, to be told what to do next ${ }^{19}$.

Cette prise de conscience qu'encourage Westworld fait écho à l'expérience de William : il aura finalement la réponse à la question posée par Logan; seulement, ce n'est pas celle qu'il espérait. Lui qui se rêvait en chevalier servant au chapeau blanc se révèle finalement être un monstre dominateur et immoral.

On ne décide pas de sa backstory, on ne décide pas de ses flashbacks, on ne décide pas du script que l'on doit suivre. Ce que l'on décide c'est ce qu'on fait à partir de tout cela. Ce que Westworld semble donc demander au spectateur c'est qu'il accepte de voir dans les personnages de fictions ses semblables, victimes comme lui d'un certain nombre de déterminismes, et qu'à partir de ce changement de perspective il fasse évoluer son rapport à la fiction. C'est donc un passage du chapeau noir au chapeau blanc ou même un passage de William à Felix, deux personnages qui semblent incarner deux niveaux de lecture opposés à travers leurs rapports aux hôtes : la fiction " pour moi » et la fiction pour les personnages qui la peuplent. C'est en cela que le moment majeur de cette première saison est peut-être celui où Maeve décide de quitter le train pour rejoindre sa fille. Pourquoi?

D'abord parce que Maeve nous montre que même si sa relation avec sa «fille » est scriptée, donc artificielle, elle existe pour elle. Elle est vraie. Ce moment fait écho à un passage de la saison 5 de la série Buffy The Vampire Slayer: des moines, cherchant à protéger le monde d'une entité néfaste nommée Glory, décident de cacher la clé permettant d'ouvrir les portes des autres dimensions en lui faisant prendre la forme d'une jeune fille, Dawn. Pour ce faire, ils créent des souvenirs artificiels pour l'héroïne de la série, Buffy, et son entourage, afin de leur faire croire que Dawn est la petite sœur de Buffy. Lorsqu'elle l'apprend, Buffy ne renie pas Dawn pour autant: si tout lui dit que Dawn est sa sœur, alors celle-ci est bien sa sœur. Si tout nous dit que les personnages de fictions sont réels, alors ils le sont. Westworld l'affirme ainsi : mes personnages sont vrais. Certes ils suivent un script, mais c'est aussi notre cas à nous, spectateurs de la série. On comprend mieux alors pourquoi le labyrinthe leur est destiné, plutôt qu'à nous. Les personnages de la série doivent se libérer, tout comme Ford et Arnold veulent libérer leurs créations. Peu importe que leur révolution soit mise en branle par des scénaristes démiurges, ce qui compte c'est son résultat : une libération. Et cette libération passe par l'improvisation.

19 L'improvisation, c'est ce moment où Maeve décide de descendre du train, allant ainsi contre son script. Ce moment de prise de contrôle est surligné par une des rares utilisations de caméra portée dans la saison, suggérant effectivement que l'on sort des 
« rails » du récit. C'est là que Maeve atteint enfin la conscience selon la pyramide d'Arnold : elle mobilise sa mémoire (le souvenir de sa fille), improvise pour son intérêt personnel et devient donc sa propre voix bicamérale, ses décisions n'étant plus dictées par un script mais par elle-même.

Nous évoquions ci-dessus deux regards sur l'improvisation: l'improvisation comme création dans un cadre prédéfini, et l'improvisation comme acte quasi divin. Ces deux perspectives se trouvent réunies dans le raga indien. Les ragas se basent sur une suite mélodique prédéterminée d'au moins cinq notes. Le musicien improvise ensuite sur cette base. Pour certains hindous, le raga serait une voie menant au moksha, c'est à dire la fin du cycle des réincarnations ${ }^{20}$. Westworld, on l'a dit, est une œuvre à forte densité musicale ; la forme musicale qui lui correspond le mieux est le raga. Westworld est partie d'une base prédéfinie : un film des années 70 , des rebondissements empruntés à d'autres séries, des archétypes; et à partir de cette base elle a constitué son récit de libération.

21 La première saison de Westworld n'était pas parfaite, c'est certain. Mais comme l'explique Ford : « Evolution forged the entirety of sentient life on this planet using only one tool: the mistake ${ }^{21}$.» En ce sens, les défauts abordés ici sont parfaitement signifiants et réflexifs : Westworld cherche ainsi à faire évoluer notre rapport à la fiction.

" I know things will work out the way they're meant to ${ }^{22}$ " annonce Dolores avant d'écraser la mouche à la fin du premier épisode, créant un lien entre cet insecte et sa foi dans l'existence d'un but à son univers. Le but de Westworld tient donc dans cette pauvre mouche. Joy et Nolan jouent ici bien sûr, sur la polysémie du mot «bug ", qui désigne à la fois un insecte et un bug informatique. Westworld est une série qui cherche à provoquer un bug, non pas informatique mais narratif. Ce bug c'est la série sans spectateur. C'est une série qui fait dérailler la machine, les rails du récit, pour en libérer les passagers. Le projet de Westworld est résumé par cette image du générique, ces mains laissant un piano jouer tout seul. Tout comme Arnold voulait que Dolores l'aide à détruire le parc Westworld, on croirait presque que les scénaristes cherchent à détruire leur série. Dans un monde conceptuellement parfait, Westworld, n'aurait pas eu de seconde saison. La délivrance des hôtes ne serait totale que si nous n'en étions pas témoins. Une autre fiction avec Ed Harris, The Truman Show, avait laissé son héros se libérer et avait fait de l'idée que le spectateur n'assiste pas à cette libération une condition sine qua non à sa réalisation.

On l'a dit, un raga est un chemin pour mettre fin au cycle des renaissances. Pour le spectateur de Westworld, cette idée rappellera le destin des hôtes qui se libèrent de leur cycle de réveils sans fin, en gagnant la conscience. Westworld semble suggérer que c'est en arrêtant la série, en les laissant improviser eux-mêmes leur destinée que nous les libérons : que le spectateur doit accepter que les personnages ne sont pas là pour l'aider à se découvrir lui-même. On attend de lui qu'il soit comme Felix, un très " mauvais humain " selon les termes de Maeve (qui ironise affectueusement ainsi sur le fait que Felix est trop humain - humane - pour un être humain "normal»). Il faudrait non seulement que les personnages puissent s'émanciper du script mais également de la caméra. Il faudrait que le piano soit sans pianiste mais également sans auditeurs. Qu'il puisse trouver sa mélodie loin de nos yeux et de nos oreilles. Ou comme le dit plus simplement Maeve : «Time to write my own fucking story ${ }^{23}$.» 


\section{NOTES}

1. https://www.lesinrocks.com/1997/08/20/actualite/ornette-coleman-et-jacques-derrida-lalangue-de-lautre-11232142/, consulté le 31 août 2018.

2. http://www2.univ-paris8.fr/deleuze/article.php3?id_article=44, consulté le 31 août 2018.

3. «Certains choisissent de voir la laideur de ce monde, sa confusion. Je choisis d'en voir la beauté. De croire qu'il existe un ordre à notre existence. Un but. »

4. http://flavorwire.com/595142/westworld-is-bullshit, consulté le 31 août 2018.

5. http://www.slate.com/blogs/browbeat/2016/12/05/ the_westworld_finale_highlighted_the_problem_with_this_show_s_twist_addiction.html? via=gdpr-consent, consulté le 31 août 2018.

6. https://uproxx.com/sepinwall/westworld-the-well-tempered-clavier-review/, consulté le 31 août 2018.

7. «Les psychothérapeutes nous disent qu'un personnage dans un rêve ne devrait pas toujours être perçu comme une personne, de la même façon que l'esprit éveillé conçoit les individus. Les personnages des rêves sont souvent des personnifications d'un aspect de la psyché du rêveur. Cela nous donne peut-être un indice quant à la raison psychologique de toute histoire. Une histoire avec des personnages fictifs utilise peut-être ces fruits de l'imagination ou de l'inconscient pour donner corps une pensée abstraite, à une idée ou à un thème. [...] On dit qu'un mythe est l'équivalent verbal d'un rite et sert le même besoin archaïque : aider l'esprit primitif à appréhender un mystère. Les histoires, même dans le contexte contemporain du divertissement de masse, seront considérées comme réussies si elles remplissent elles aussi ce besoin, ce dont les spectateurs n'ont même pas besoin d'être conscients. » Alexander Mackendrick, On Filmmaking [2004], New York, Farrar, Straus and Giroux, 2005, p. 10-11. Notre traduction.

8. «As-tu déjà remis en question la nature de ta réalité ?»

9. «De nos jours nous sommes identiques.»

10. "Cet endroit est la réponse à la question que tu te poses : qui es-tu vraiment?»

11. De la même façon, lors du visionnage, chaque spectateur fait l'expérience d'une relation unique avec Dolores (ou tout autre personnage de la série), relation qu'il partage pourtant avec le reste du public.

12. «Vous m'avez construite pour prédire les actes des gens, Harry. Mais pour les prédire, il faut les comprendre et cela s'est avéré très difficile. J'ai commencé par diviser leurs vies en moments, en cherchant les connexions, ce qui explique qu'ils aient fait ce qu'ils ont fait »

13. «Ils peuvent vous faire oublier... mais apparemment, cela ne s'applique pas à toi. »

14. Jorge Luis Borges, Fictions, Paris, Gallimard, 2015, p. 75.

15. «Comment peux-tu en être sûr?»

16. «Chaque hôte a besoin d'une backstory, Bernard. Vous le savez très bien. L'identité est une sorte de fiction, pour les hôtes comme pour les humains.»

17. C'est la dimension marxiste de Westworld.

18. «Quelle est la différence entre ma souffrance et la vôtre, entre vous et moi ?»

19. "C'est précisément cette question qui obsédait Arnold, le faisait se sentir coupable, et qui l'aura rendu fou. La réponse m'a toujours semblé évidente: il n'existe pas de seuil à dépasser pour que la totalité dépasse la somme des parties. Pas de point d'inflexion à dépasser pour devenir vivant. On ne peut définir la conscience parce qu'elle est indéfinissable. Les humains s'imaginent qu'il y a quelque chose de spécial dans notre façon de voir le monde et pourtant nous vivons dans des boucles aussi étroites et fermées que celles des hôtes. Nous remettons rarement 
en question nos propres choix, et acceptons sans difficulté, dans l'ensemble, que l'on nous commande quoi faire. »

20. William Forde Thompson, Music in the Social and Behavioral Sciences: An Encyclopedia, Londres, SAGE, 2014, vol. 2, p. 951.

21. "L'évolution a forgé la totalité de la vie consciente sur cette planète à l'aide d'un seul outil : l'erreur.»

22. "Je sais que tout se passera comme prévu.»

23. «Il est temps d'écrire ma propre putain d'histoire. »

\section{RÉSUMÉS}

Cet article s'intéresse à la réception critique et populaire de la première saison de la série Westworld. Il explore la façon dont les éléments qui ont été perçus comme des défauts par une partie du public peuvent en réalité être interprétés comme étant en parfaite adéquation avec les préoccupations thématiques de la série. Cette réflexion mène à une analyse du principal problème qui semble préoccuper Westworld : la libération de ses personnages.

This paper deals with the critical and popular reception of the first season of the TV series Westworld. It explores how the elements that have been perceived as flaws by a part of the audience can in fact be interpreted as being perfectly coherent with the thematic preoccupations of the series. This reflection leads to an analysis of what appears to be the main issue in Westworld : the liberation of its characters.

INDEX

Mots-clés : Personnages, improvisation, Borges, libération, posthumain, spectateurs, Westworld Keywords : Characters, improvisation, Borges, liberation, posthuman, viewers, Westworld

\section{AUTEUR}

\section{BRIAC PICART-HELLEC}

Briac Picart-Hellec rédige actuellement une thèse inscrite à l'Université Le Havre Normandie sur la série Twin Peaks. Il a travaillé sur Les Soprano et Deadwood et présenté des communications sur Twin Peaks, Lost et Westworld. Parallèlement à ses recherches il réalise des courts-métrages. Briac Picart-Hellec is currently writing a doctoral thesis at University Le Havre Normandie on the series Twin Peaks. He has worked on The Sopranos and Deadwood and he has given presentations on Twin Peaks, Lost and Westworld. Concurrently with his studies, he has directed a number of shortfeature films. 